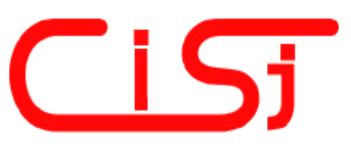

\title{
EVOLUTIONARY DESIGN OF WIND TURBINE BLADES
}

\author{
V. Díaz Casás, R. J. Duro, F. López-Peña \\ Integrated Group for Engineering, University of A Coruña (Spain), \\ vdiaz@udc.es, richard@udc.es, flop@udc.es,www.gii.udc.es
}

\begin{abstract}
An automatic design environment is implemented for the aerodynamic design of wind turbine blades. This tool involves the integration of evolutionary techniques and a simple, fast, and robust aerodynamic simulator which was developed for the prediction of the performance of any turbine blade produced by the evolutionary process. The aerodynamic simulator is based on blade element theory in which a panel method is combined with an integral boundary layer code to calculate the blade airfoils' characteristics. In order to reduce computations some simplifications have been applied and the results corrected by means of the application of neural network based approximations. Results of the simulations obtained using this technique, of the application of the automatic design procedure and of the operation of the wind turbines thus obtained are presented.
\end{abstract}

Keywords: Wind Turbines, Evolutionary Techniques, Automatic.

\section{INTRODUCTION}

To obtain efficient wind turbine blades that lead to acceptable economical performance of the turbines during their lifetime involves a complex aerodynamic design process. It is clear that any improvement in the aerodynamic design of wind turbines implies a significant profit increase during the 20 years they will be operating. This paper proposes an approach to carry out this task by means of evolutionary techniques within an automatic design environment. For this purpose, a simple, fast, and robust aerodynamic simulator has been developed and embedded within a design environment. The simulator is able to predict the performance of any turbine produced as an intermediate individual of the evolutionary process. The aerodynamic simulator is based on blade element theory in which an airfoil analysis method based on potential theory is combined with an integral boundary layer code in order to calculate the aerodynamic characteristics of blade airfoils when accounting for viscous effects. In order to reduce computational cost, some simplifications are contemplated in the airfoil performance estimation and the results corrected by means of the application of neural network based approximations. The evolutionary design environment approximation to the design problem is based on work carried out by other authors in the application of evolution for the design and optimization of different types of systems in other realms. Examples of applications in this line are described by Kirsch and Rozvany for the structural optimization of bridges and transmission towers [1], by Keane et al. [2], Song et al. [3] or Ong et al. [4] in airplane and airfoil design optimization.

\section{DESIGN ENVIRONMENT}

A design environment based on evolutionary techniques was created with the objective of achieving valid results in generic optimization or design processes. This has led to its implementation as a set of independent blocks which can be substituted and relinked depending on the particular design process under consideration and the types of simulations and evaluations it requires to achieve the reliability and characteristics expressed in the specification stage.

The basic structure of the design system comprises three blocks:

- Solution search block.

- Decision making block (evaluation with or without human participation).

- Computational distribution block.

The search stage is based on different types of evolutionary algorithms employing a decision block for the evaluation of candidate solutions. The algorithm initially chosen for the design of wind turbine blades is a standard macroevolutionary algorithm. The reason for this choice is that the emphasis of the work was on evaluating the 
operation of the system and this could be achieved more easily through the standard macroevolutionary algorithm. This algorithm uses a decision module based on a blade simulator that was developed ad hoc for the evaluation of each possible candidate. This simulator provides the fitness value needed for evolution to be carried out.

Several subsystems are included in the environment by means of standard interfaces allowing it to fit many different design problems. In the present case, the design is not aimed at the improvement of an initial model; on the contrary, we want to generate a completely new blade model. This approach makes it necessary to develop a structure that addresses all the stages of the design process. Furthermore, one of the objectives is to reduce human participation as much as possible. As a general rule, human participation should be limited to the mere definition of the problem. As it is common place in any design process, the initial constraints generate a space of very high, and usually variable, dimensionality, but at least in the present case the search path is not conditioned by fictitious constraints which could be introduced by an arbitrary behavior of the human designer due to his/her level of experience and complex problem solving skills.

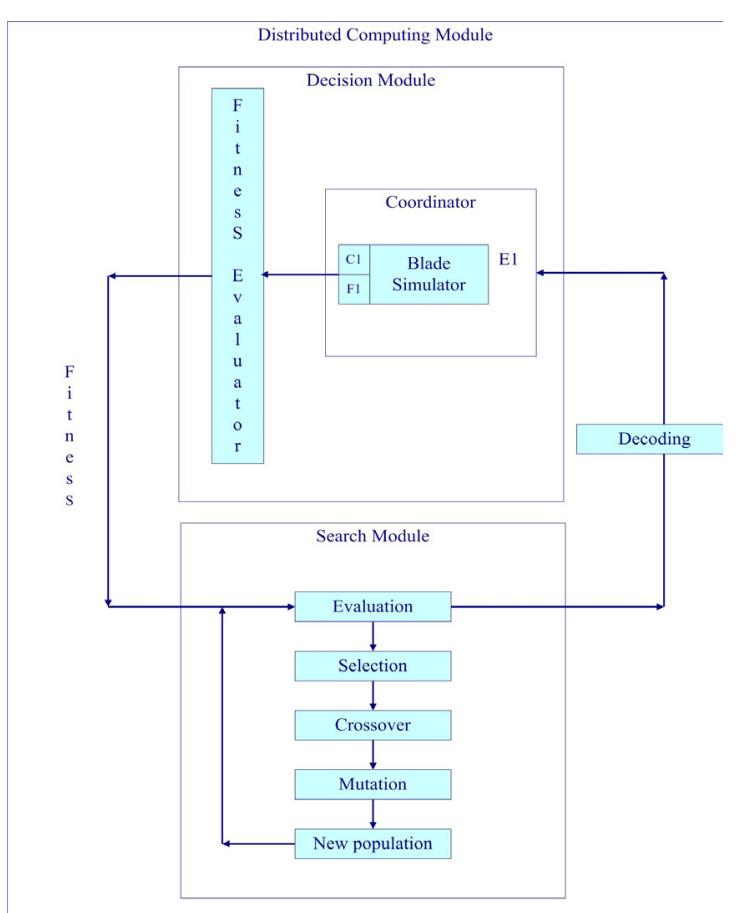

Fig. 1 - Environment design Structure

\section{SEARCH FOR SOLUTIONS}

As in any other design problem the goal here is to achieve the optimum value for a fitness function representing the objective sought in the process. In this work, the fitness function is obtained from the application of the simulator described in section 4 and presents discontinuities and ungrouped local maxima surrounded by flat regions produced by non-valid individuals. This is a very difficult fitness landscape to explore through traditional methods and even standard genetic algorithms due to the lack of information provided by the flat regions and the many local maxima. In order to improve the search, a basic marcroevolutionary [5] algorithm has been implemented, providing for a more efficient exploration in this type of search spaces as shown by Becerra et al. [6].

The adopted search procedure is an extension of evolutionary techniques to the evolution of species, where non-adapted species become extinct and are replaced by new species. The criterion to decide whether a species becomes extinct can be stated as

$$
\sum_{j} \frac{q_{i}-q_{j}}{\left|x_{i}-x_{j}\right|}<0
$$

Where $q_{j}$ is the fitness of the each individual and $\left|\mathrm{x}_{\mathrm{i}}-\mathrm{x}_{\mathrm{j}}\right|$ is the Euclidean distance between the two chromosomes.

The strategy for the generation of a new individual depends parameter $\tau$. In the first half of the evolution process its values grows linearly from zero to one and, in the second half, its value remains constant. Each species that disappears is replaced by a new random individual if a random value is larger than $\tau$, or by an individual resulting from the interpolation of the extinguished one and a random one from the remaining population otherwise.

To further improve performance and reduce the risk of a population converging to local optima, the process is run for more than one population simultaneously; each subpopulation is called a race. The communications between different races is carried out through migrations. A migration implies that the best individual in each race is included in the populations of the other races. This migration processes may take place among neighboring populations (local migrations) or among all the races (global migrations).

Thus, using these strategies, two phases can be distinguished during the evolution process: a first phase where the main process is the exploration of the solution or search space and a second phase where the main process taking place is the exploitation of interesting candidate areas.

\section{AERODYNAMIC SIMULATOR}

The decision module in the automatic design process is made up of an aerodynamics simulator for blades. This module is the one that provides a fitness 
value for every individual in every population of the evolutionary process. To reduce computation time a specific simulator based on the traditional blade element theory has been developed. The method is able to perform a fast evaluation of a blade through the partition of the blade into blade elements allowing it to obtain the blade's efficiency through the study of each section. These values are corrected through the observation of the effects generated on a given section by the surrounding sections, as well as the rotating wake.

Starting from this formulation one can obtain a productive relationship between the apparent incident wind and that induced on the blade itself by the vortices developing downstream in its wake. From this, the actual angle of attack of every blade airfoil can be determined. This effective angle of attack distribution is needed to calculate lift and drag forces acting on each blade section, and thus to obtain the torque produced by the blade on the shaft [7].

From basic fluid mechanic analysis it can be proven that, under wind mill working conditions, the flow behaves as ideal. In aerodynamics the studies of forces acting on a body immersed in an ideal fluid are carried out by solving Euler's equations of motion. However, this method only allows for the extraction of pressure forces but not for friction. In a real situation there are certain regions near the solid surface, the boundary layers, where viscous phenomena become dominant. Therefore, to obtain the frictional forces applied to the body, it is necessary to solve the boundary layer equations around its surface. In addition, in many cases the boundary layer detaches from the surface, and this modifies the pressure distribution obtained from Euler equations.

With these considerations in mind, the process of calculating the characteristics of the airfoil has been divided into three stages:

- Analysis of the flow around the airfoil considering it an ideal fluid.

- Study of the boundary layer.

- Application, if necessary, of the correction for detachment of the boundary layer.

\section{POTENTIAL FLOW ANALYSIS}

To obtain a first approximation of the air velocity distribution around the airfoil, air is taken as an uncompressible perfect fluid. With these considerations the velocity field can be calculated as a gradient of a scalar function. Thus, this function is defined by the boundary conditions of the airfoil.

$$
\Delta \Phi=0 \quad \vec{V}=\vec{\nabla} \Phi
$$

To calculate forces over the airfoil, pressure forces are evaluated applying the Benoulli theorem.

$$
\begin{gathered}
\frac{\partial \Phi}{\partial t}+\frac{1}{2} \vec{V}^{2}+\frac{p}{\rho}=k \\
\vec{F}=-\int p £ d A=\rho \int \frac{\partial \Phi}{\partial t} \AA d A+\frac{1}{2} \rho \int \vec{V}^{2} \mathrm{E} d A
\end{gathered}
$$

If $U$ is the velocity of incidental air, then

$$
\frac{\partial \Phi}{\partial t}=\frac{d \vec{U}}{d t} \Phi-\vec{U} \cdot \nabla \Phi=\frac{d \vec{U}}{d t} \Phi-\vec{U} \cdot \vec{V}
$$

In steady motion $U$ is constant, and the acting forces are

$$
F_{i}=\rho \int\left(\frac{1}{2} \vec{V}^{2}-U_{j} \cdot V_{j}\right) n_{i} d A
$$

With $n$ as the outward normal to the body surfaces and since $V$ is irotational, we have

$$
\begin{aligned}
& \frac{1}{2} \int \vec{V}^{2} n_{i} d A=U_{j} \int V_{i} n_{j} d A \\
& F_{i}=\rho U_{j} \int\left(V_{i} \cdot n_{j}-V_{j} \cdot n_{i}\right) d A
\end{aligned}
$$

\section{BOUNDARY LAYER EFFECTS}

Considering a potential flow, viscous forces were neglected. Consequently, an important component of drag forces was not taken into account. To solve this problem it is necessary to add corrections that introduced the effect of viscous processes appearing in the boundary layer. Two models are considered in this analysis:

- Thwaites' method in laminar cases [8].

- Head's method in turbulent cases [9].

These methods are based on Von Karman's integral equation, which, in the case of an uncompressible and two-dimensional boundary layer, can be written as

$$
\begin{gathered}
H=\frac{\delta^{*}}{\theta} \quad C_{f}=\frac{\tau_{f}}{\frac{1}{2} \rho U_{e}^{2}} \\
\frac{d \vec{V}}{d t}+\frac{1}{U_{e}} \frac{d U_{e}}{d x} \theta(2+H)=\frac{C_{f}}{2}
\end{gathered}
$$

Von Karman's equation contains three unknown variables defining the boundary layer: displacement thickness $\left(\delta^{*}\right)$, momentum thickness $(\Theta)$, and drag stress $\left(C_{f}\right) . U_{e}$ is the flow velocity previously 
obtained at the body surface by the potential flow analysis. To solve the equation it is necessary to use methods that allow a reduction of the number of parameters.

\section{THAWAITES' AND HEAD'S METHODS}

Thwaites' method starts with the nondimensional form of Von Karman's equation

$$
\frac{U_{e} \cdot \theta \cdot d \theta}{v \cdot d x}+\frac{\theta^{2}}{v} \frac{d U_{e}}{d x}(2+H)=\frac{\tau_{f} \theta}{\rho U}
$$

Where the parameter $H$ is defined as:

$$
H=\frac{\delta-\delta^{*}}{\theta}
$$

Then a new parameter $\lambda$ is introduced:

$$
\lambda=\frac{\theta^{2}}{v} \frac{d U_{e}}{d s}
$$

Thus the integral equation can be expressed as:

$$
\begin{aligned}
& U_{e} \frac{d}{d x} \frac{\lambda}{\frac{d U}{d x}}=2\left(\frac{\tau_{f} \theta}{\rho U_{e}}-\lambda(H(\lambda)+2)\right)= \\
& =2(S(\lambda)-\lambda(H(\lambda)+2))=F(\lambda)
\end{aligned}
$$

Thwaites combined analytical and experimental results to achieve an explicit definition of function $\mathrm{F}$,

$$
F(\lambda)=0.45-6 \lambda
$$

Using this function, the previous equation can be integrated to obtain:

$$
\theta^{2}=\frac{0.45 \cdot v}{U_{e}^{6}(x)} \int_{0}^{x} U_{e}^{5}(x) d x
$$

With these results, the friction coefficient can be calculated for each point as:

$$
C_{f}=\frac{2 \mu}{\rho U_{e} \theta} S(\lambda)
$$

When turbulence effects become significant, the accuracy of Thwaites' method decreases. This makes it necessary to use other functional forms; in this case we have selected Head's function. By empirical considerations it stands that in a turbulent boundary layer the friction coefficient can be evaluated as:

$$
C_{f}=0.246 \cdot\left(10^{-0.678 H}\right) \operatorname{Re}_{\theta}^{-0.268}
$$

\section{CORRECTION BY NEURAL NETWORK}

Calculations of drag and lift forces by potential flow plus boundary layer theories result in good accuracy in cases where the boundary layer remains attached to the body or if it detaches close to the airfoil's trailing edge. Nevertheless, the range of angles in which it is necessary to carry out the study of the airfoil demands analyzing positions where the detachment corresponds to a large part of the airfoil's surface. In these configurations the error of the calculations based on potential flow is high. To correct the deviations that appear when the detachment of the boundary layer takes place at a position below $90 \%$ of the chord, a neural net was used. This is a genuine novelty of present work.

Two simple multilayer perceptron neural networks with 6 input neurons (Reynolds number, angle of attack, lift coefficient, drag coefficient, \% upper detachment, \% lower detachment), two hidden layers of 6 neurons each and 1 output neuron (Lift coefficient or drag coefficient) were employed. A backpropagation algorithm was applied to obtain the weights of the synapses (training process) using a set of 110 real data. These data were obtained from real analyses of NACA 2410, NACA 0009, NACA 2415, NACA 2541, CLARKY, ClarkySM and DAE11 airfoils [10].

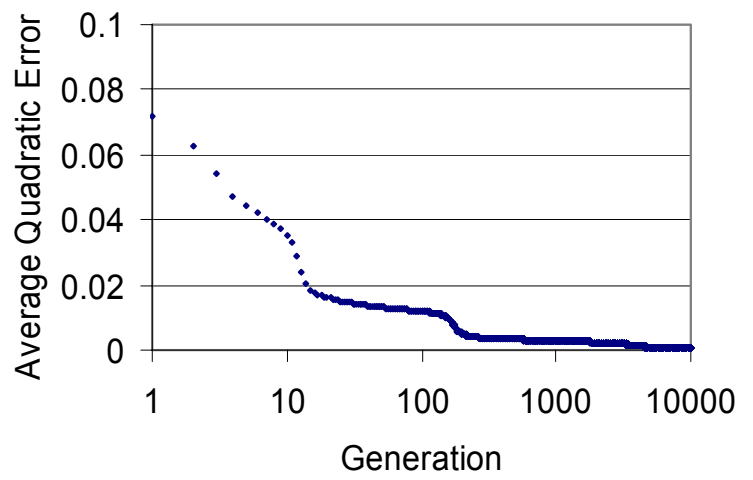

\section{Fig. 2 - Evolution of average quadratic error} during network training

After 10000 training steps an average quadratic error of around $0.6 \%$ was obtained for the test samples, showing that this kind of mathematical approach is a very appropriate technique for our problem. The use of neural net corrections implied a significant increase in the accuracy of the method; the average quadratic relative error is reduced from $12.4 \%$ to $0.6 \%$ without a significant increase in the calculation time. Any known method able to correct for boundary layer detachment effects would be extremely costly in terms of computation. 


\section{D INTEGRATION}

The effective power that each blade can produce was calculated as the integral of the effects of each airfoil. To extrapolate 2D airfoil results to 3D blade elements, it was necessary to introduce the effect of fluid circulation around the blade in the calculation procedure. To introduce this effect a Prandtl correction was used [11]. In addition, the attack angle of each airfoil was calculated as the angle that obtains the optimal drag-lift relation.

\section{EXPERIMENT}

The first step, in order to solve a design problem, is determining the real conditions of the problem and the parameters required to define every individual. As a particular test problem, the environment developed is being used in designing a windmill blade having a maximum diameter of $16 \mathrm{~m}$. and an average wind speed operation condition of $7 \mathrm{~m} / \mathrm{s}$ with a Weibull's distribution. A theoretical maximum power, called the Betz limit [12], can be easily calculated for any windmill; this is used as a control parameter for the fitness function. The Betz limit can never be achieved in reality, but it is useful as a measure of the quality or fitness of a blade by comparison.

Any blade geometry is defined by providing its distributions of chord, twist and airfoil shape along the span. In this case we have settled for thirty sections, considering the rest of the sections as interpolations from these. The NACA four digit family of airfoils was used in order to define the blade sections. This family of airfoils permits the complete definition of their shape through just four parameters. Regarding the distribution of twist and chord along the blade, they are established after setting an initial value in a given root position near the mill shaft. In this case, the initial chord value is set at $8 \%$ of the windmill's radius. Finally, the last design parameter is the angular velocity of the wind turbine, which is determined by means of the so called lambda parameter (ratio between the velocities of the blade tip and wind).

Each blade is thus encoded in the evolutionary process by a chromosome made up of one hundred and five genes. The range of initial values is:

- NACA profiles: 0000 to 6619

- Angle: -25 to 25 degrees

- Chord: 0.2 to 1.3 .

For the remaining sections of the blade, the parameters are defined as a modification of the previous section. The twist value is increased by a value between $\pm 3^{\circ}$, and the chord value is modified by a percentage of up to $5 \%$

\section{Best Individual Fitness}

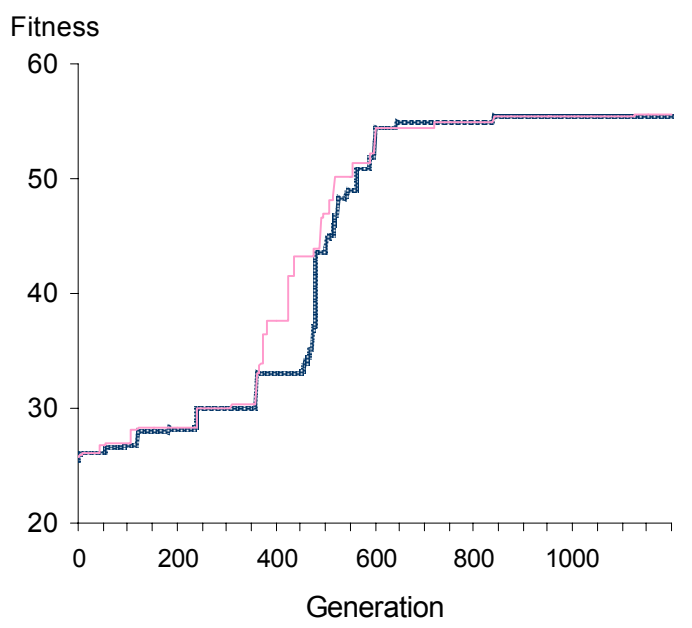

Fig. 3-Comparison between the fitness of the best individual in the best and worst race each generation

In this study, structural forces and design are not considered. However chord constraints are introduced in order to achieve the possibility of having a real (manufacturable) blade chord distribution, for this reason, only decreasing distributions are taken into account.

As commented before, the analysis of the fitness landscape generated by the fitness function has made us resort to a macroevolutionary algorithm. The results, presented in this paper were obtained with a population of 640 individuals distributed into 8 races.

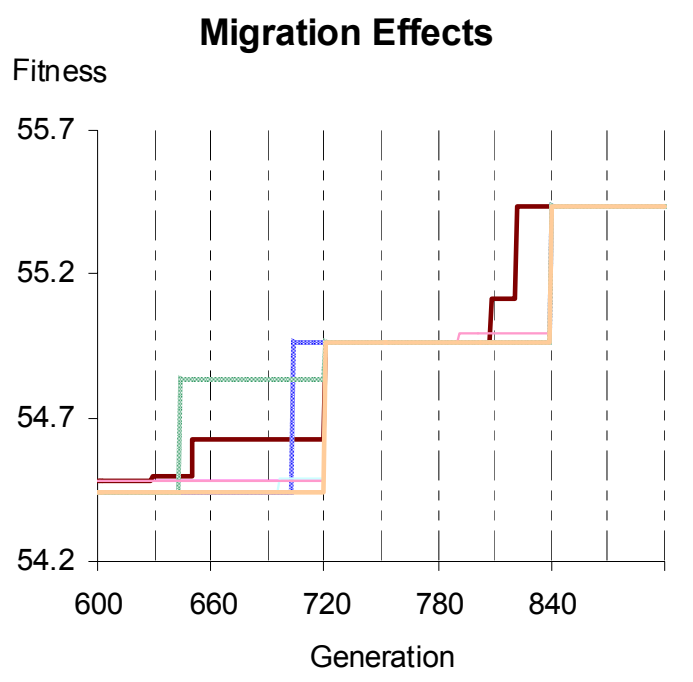

Fig. 4 - Global and local migration effects in the fitness of the best individual in each race. Corresponds to an enlarged area of figure 3.

The final blade was obtained in the 1200th generation. Fig. 3 and Fig. 4 display the evolution of the maximum value of the fitness for each 
generation and the effects of migrations on the evolution process. In this experiment, local and global migrations are considered. Local migrations take place every sixty generations and global migrations every one hundred and twenty generations. Mechanical performance and other simplifications included in the simulator induce some errors in the numeric result, but do not affect the comparisons between blade shapes.

Fig. 5 displays the shape of the best blade obtained for the conditions stated above. This shape was achieved in the 1200th generation and its efficiency is $71.6 \%$.

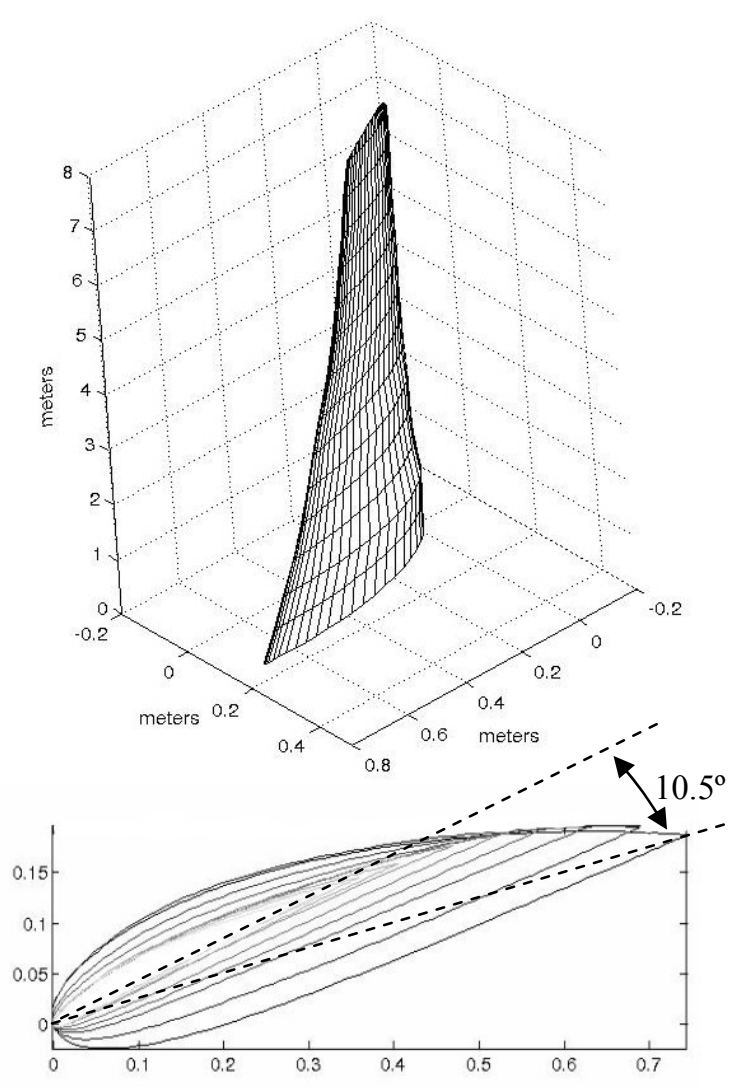

Fig. 5 - Top and Isometric view of the best individual achieved in this experiment

\section{CONCLUSION}

This paper deals with de construction of an automatic design environment for windmill blades linked to the development of an aerodynamic simulation strategy for determining the fitness of each blade. This simulator firstly computes the characteristics of any blade's section profile through potential flow methods combined with boundary layer theory. Then results are corrected by a novel method based on the implementation a neural network to account for effects having to do with detachment of the flow. After obtaining the characteristics of all the blade sections, the simulator computes the performance of the whole blade through the application of the classical blade element method.

The results obtained in a test case are presented as an example. The results obtained in this or in any other case are very precise and clearly meet all of the design parameters imposed without having to resort to very complicated and costly non linear models of the plant within the simulator. In the tests of the design environment, taking some particular conditions into account, near optimal results for the blade were achieved.

This work was carried out as a first step in the construction of an integrated automatic design environment. It establishes an open base where evolutionary operators and simulators can be easily changed to handle many different design problems.

\section{ACKNOWLEDGEMENTS}

This work was funded by the Xunta de Galicia under projects PGIDIT-03REM08E and PGIDIT04DPI166004PR.

\section{REFERENCES}

[1]. Kirsch, U. \& Rozvany, G.I.N.: "Alternative formulations of structural optimization". Structural Optimization. 7, 1994, pp. 32-41

[2]. S. A. J. Keane, "Wing Optimization Using Design of Experiment, Response Surface, and Data Fusion Methods", J. Aircraft 40(4),(2003).pp.741-750

[3]. W. Song, A. J. Keane, M. H. Eres, G. E. Pound, and S. J. Cox, "Two Dimensional Airfoil Optimisation using CFD in a Grid Computing Envirionment" in Proc. Euro-Par 2003, Klagenfurt (2003)., pp. 50-57

[4]. Y.S. Ong, P.B. Nair, A.J. Keane, and K.C. Wong, "Surrogate-Assisted Evolutionary Optimization Frameworks for High-Fidelity Engineering Design Problems", pp. 307-331 in Knowledge Incorporation in Evolutionary Computation: Studies in Fuzziness and Soft Computing, Vol. 167, ed. Yaochu Jin,SpringerVerlag, (2004).

[5]. Marín, J., Solé, R.V. (1999), "Macroevolutionary Algorithms: A New Optimization Method on Fitness Landscapes", IEEE Transactions on Evolutionary Computation 3(4):272-286

[6]. J.A. Becerra et al. "MA vs. GA inn Low Population Evolutionary Processes with Mostly Flat Fitness Landscapaes". Proc. 6th Joint Conference on Information Sciences -2002, 
Research Triangle Park. North Carolina. (2003) Pp 626-630.

[7]. Martin O. L. Hansen, "Aerodynamics of Wind Turbines", Science Publishers. Ltd, 2000, pp.11-66.

[8]. Thwaites, B. "On the momentum equation in laminar boundary layer flow". ARC RM 2587. 1952.

[9]. Head, M. R. "Entrainment in the turbulent boundary layers". ARC R\&M 3152, 1958

[10]. John Moores, Potential Flow 2-Dimensional Vortex Panel Model: Applications to Wingmills, Master's thesis. University of Toronto. Faculty of Applied Science and Engineering. April 2003.

[11]. Jones K.D., Davids, S. and Platzaer. "Oscilation Wing Power Generation" ASME paper 99-7050 in Proceedings of the thirds ASME/JSME Joint Fluids Engineering Conference.

[12]. Abbot, H. and Von Doenhoff, Albert E, "Theory of Wing Sections". Dover (1949; reprinted 1958).

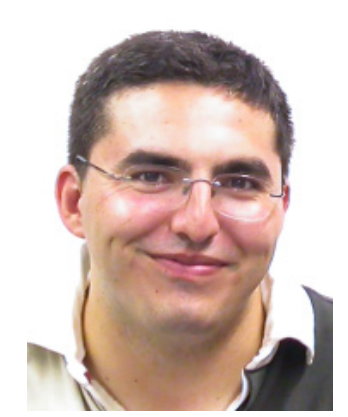

Vicente Díaz Casás, received a M.S. degree in Naval and Oceanic Engineering from the University of A Coruña, Spain in 2004. Since 2004 he has worked in Fluid Engineering Group of the University of $A$ Coruña. His research interests include aerodynamic sail design and underwater unnamed vehicles design.

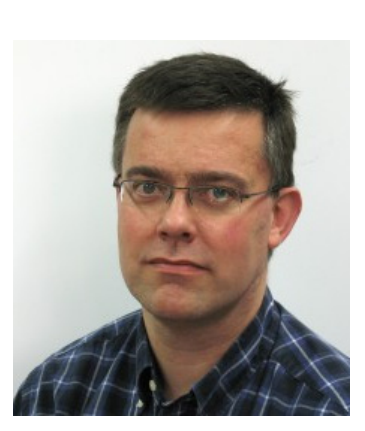

Richard J. Duro received the B.S. and M.S. degree in Physics from the University of Santiago de Compostela, Spain, in 1989, and a PhD in Physics from the same University in 1992. He is currently a "Profesor Titular" in the Department of Computer Science and member of the Autonomous Systems Group at the University of A Coruña, Spain. His research interests include higher order neural network structures, signal processing and autonomous and evolutionary robotics.

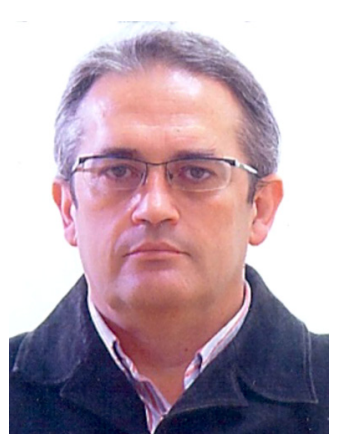

Fernando López Peña is "Profesor Titular" in the Naval and Oceanic Engineering Department and member of the Fluid Engineering Group at the University of A Coruña, Spain. He received his MS degree as Aeronautical Engineer in 1981 from the Polytechnic University of Madrid, Spain, and his PhD in 1992 from the University of Louvain, Belgium. Current research activities are related to hydrodynamics and aerodynamics of bodies and vehicles, development of instruments for flow measurement and diagnosis, and signal and image processing. 